\title{
Empirical Estimation of Virtual Point Source Height Over a Bank of Coke Ovens
}

\author{
Jacek Żeliński $^{1}$ • Dorota Kaleta ${ }^{2}$ • Jolanta Telenga-Kopyczyńska ${ }^{1}$
}

Received: 21 May 2015 / Accepted: 4 May 2016/Published online: 19 May 2016

(C) The Author(s) 2016. This article is published with open access at Springerlink.com

\begin{abstract}
This paper addresses an empirical method of assessment of the height of a virtual point source. In order to achieve such assessment, three planes defining the location of a virtual point source in a space are created and formulas describing the geometry of observer's sight and plume orientation are used. Data for calculations are obtained on a basis of plume photographs. The final position of a virtual emission point is described by a set of its coordinates in the Cartesian coordinate system. Presented method may be applied as a means to verify and validate the existing formulas describing the rise of plume above the stack exit or as a tool enabling acquisition of information for the sake of creating new formulas of this type. The calculation of the location of a virtual point source ascribed to the point emission from the roof of the bank of coke oven is presented and illustrated with photographs which reveal position of plume, adequate planes, axes and characteristic objects.
\end{abstract}

Keywords Virtual point source $\cdot$ Plume rise $\cdot$ Pollution dispersion modelling $\cdot$ Height of emission point

\section{Introduction}

Calculations of pollutant dispersion in the atmospheric air, conducted by application of Gaussian plume models, comprise two main stages:

Jacek Żeliński

jzelinski@ichpw.pl

1 Institute for Chemical Processing of Coal, Zamkowa 1, 41-803 Zabrze, Poland

2 Department of Air Protection, Silesian University of Technology, Konarskiego 22B, 44-100 Gliwice, Poland
- Calculating the position of what is referred to as a virtual point source (VPS), usually limited to the point's altitude above ground level

- Calculating propagation of pollutants introduced at this particular point into the atmospheric air

Specifying of the VPS are mainly envisaged to determine the altitude of a hypothetical point at which, as it is assumed, the pollutants being emitted are discharged into the air, although calculations may cover all three coordinates of such point. This altitude is a sum of the stack height and the plume rise- the vertical distance the pollutants travel after leaving the stack before taking direction of motion parallel to the ground surface $[1,2]$.

Determination of the actual plume rise is difficult - computational values obtained in practice are usually encumbered with considerable errors. For purposes of dispersion calculations, the plume rise is typically achieved by:

- Applying empirical formulas [3]

- Solving equations of mass, momentum and energy conservation describing the motion of a waste gas stream after leaving the stack exit [4]

- Observing the plume, also by means of a plume contrasting markers or thermal imaging cameras for hot gases [5]

This paper describes an empirical method of assessing a VPS position against the coordinate system assumed. The means applied for this purpose include plume photographs used to analyse its trajectory against characteristic terrain points around the facility and formulas describing the geometry of planes defining the $V P S$. The method in question was devised for verification of computational formulas applied to assess the VPS altitude, especially in cases of non-typical objects, such as a bank of coke ovens, characterised by heat emission above the average. 


\section{Materials and Methods}

The VPS altitude $(H)$ is assumed to be a vertical coordinate of a point positioned on the plume axis in a place where the ultimate altitude of its trajectory has been obtained. Coordinates of the $V P S$ position are estimated based on calculations conducted under the assumption, that the investigated point is created by intersection of three planes (Fig. 1). Plane 1 is the observer's sight plane sloping towards the base, plane 2 is the observer's vertical sight plane, plane 3-the vertical plane in which the plume moves in the wind direction.

While analysing the arrangement of planes, one applies the Cartesian coordinate system with axis $X$ indicating the east, axis $Y$ indicating the north and $Z$ being the axis of altitude.

In order to find the VPS coordinates, one must solve a system of three general equations of plane of the following type:

$A_{n} x+B_{n} y+C_{n} z=D_{n}$

describing spatial orientation of plane $1 \div 3[6,7]$, where $n=1$, 2, 3 .

From mathematical point of view, developing the general equation for each of the planes can be brought down to finding components of any two vectors located on the given plane. These vectors may be established based on coordinates of three points of the plane (with the vectors typically sharing a point corresponding to the beginning or the end of each of them). Next, one applies the vector product to calculate components of the given plane's normal vector. It corresponds to finding coefficients $A_{n}, B_{n}$ and $C_{n}$ of Eq. (1) of the $n$th plane. Finding coefficient $D_{n}$ consists in solving Eq. (1) at any point of known coordinates, positioned in the $n$th plane. The plume orientation observations required to conduct the calculations and the estimation of the plume dimensions are based on the photographs which enable the length of sections corresponding to apparent plume dimensions to be measured as perceived by the observer. It is them that provide grounds for calculation of actual dimensions and orientation of the plume. Planes $1 \div 3$, used to find the $V P S$, are developed in the following manner.

\subsection{Plane 1}

Plane 1 is the observer's sight plane sloping towards the base (Fig. 2). The plane intersects with the land surface at the angle of $\delta$. Angle $\delta$ depends on the VPS altitude above ground level as well as its horizontal distance from the observation point (Pobs). In reality, a natural line of the observer's perception does not form plane 1 but a side surface of a cone with the vertex at the observation point. In order to obtain the intended effect of observation along plane 1, one projects the VPS altitude being observed on the apparent axis of altitude in a direction parallel to the upper edge of a properly selected reference object. The observation point should be chosen very thoroughly.

In order to increase the accuracy of finding the ultimate altitude of plume, the angle between planes 3 and 2 (assuming counter-clockwise direction of an angle, Fig. 1) should be as close as possible to, and not greater than, $90^{\circ}$.

The VPS altitude being observed is assessed by reading it at the apparent altitude axis (point Paref) with the scale established by way of comparison between the height of a nearby reference object of known dimensions and the length
Fig. 1 Observer's sight planes 1 and 2 and plume motion plane 3

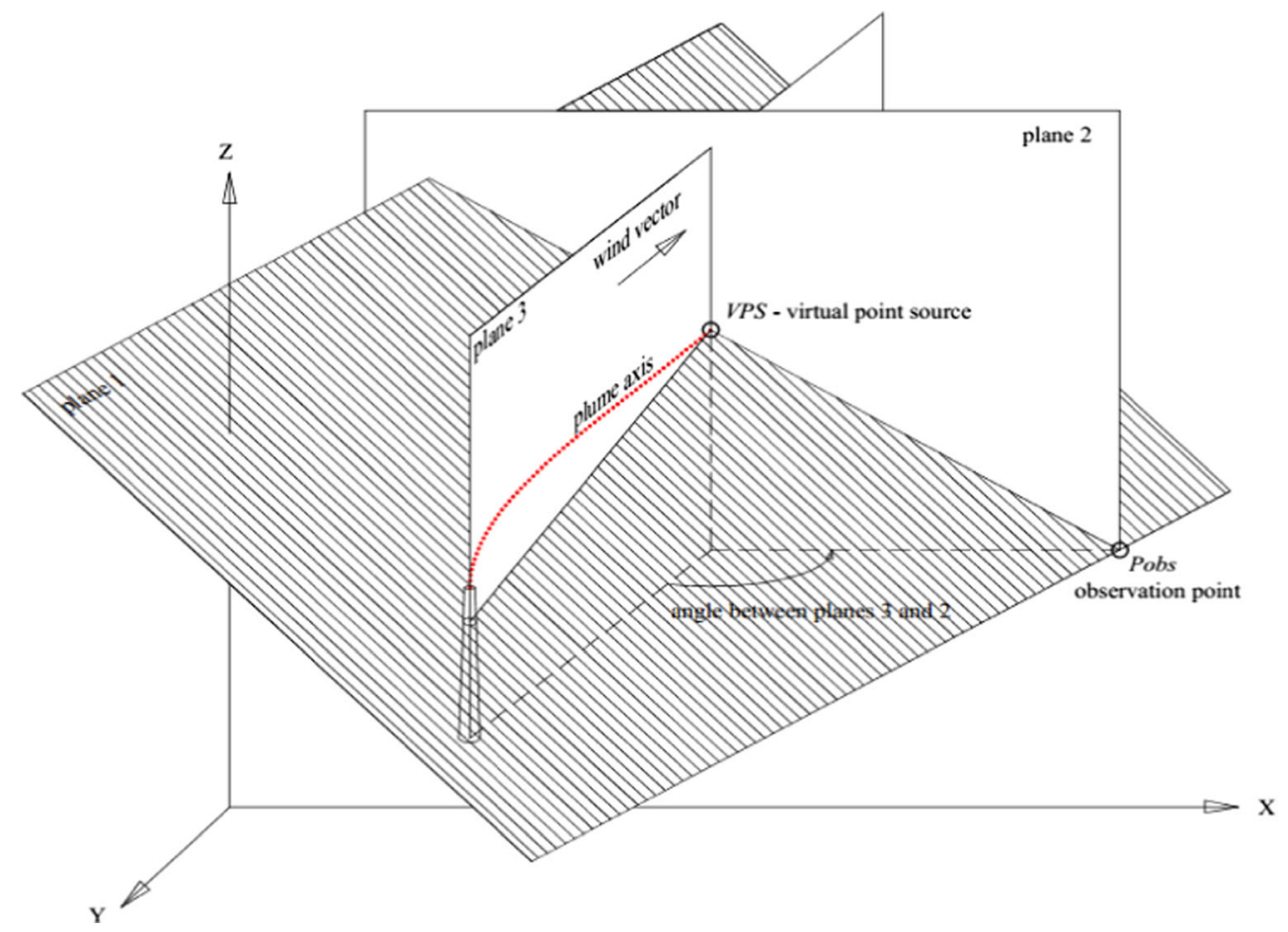


Fig. 2 Orientation and layout of plane 1

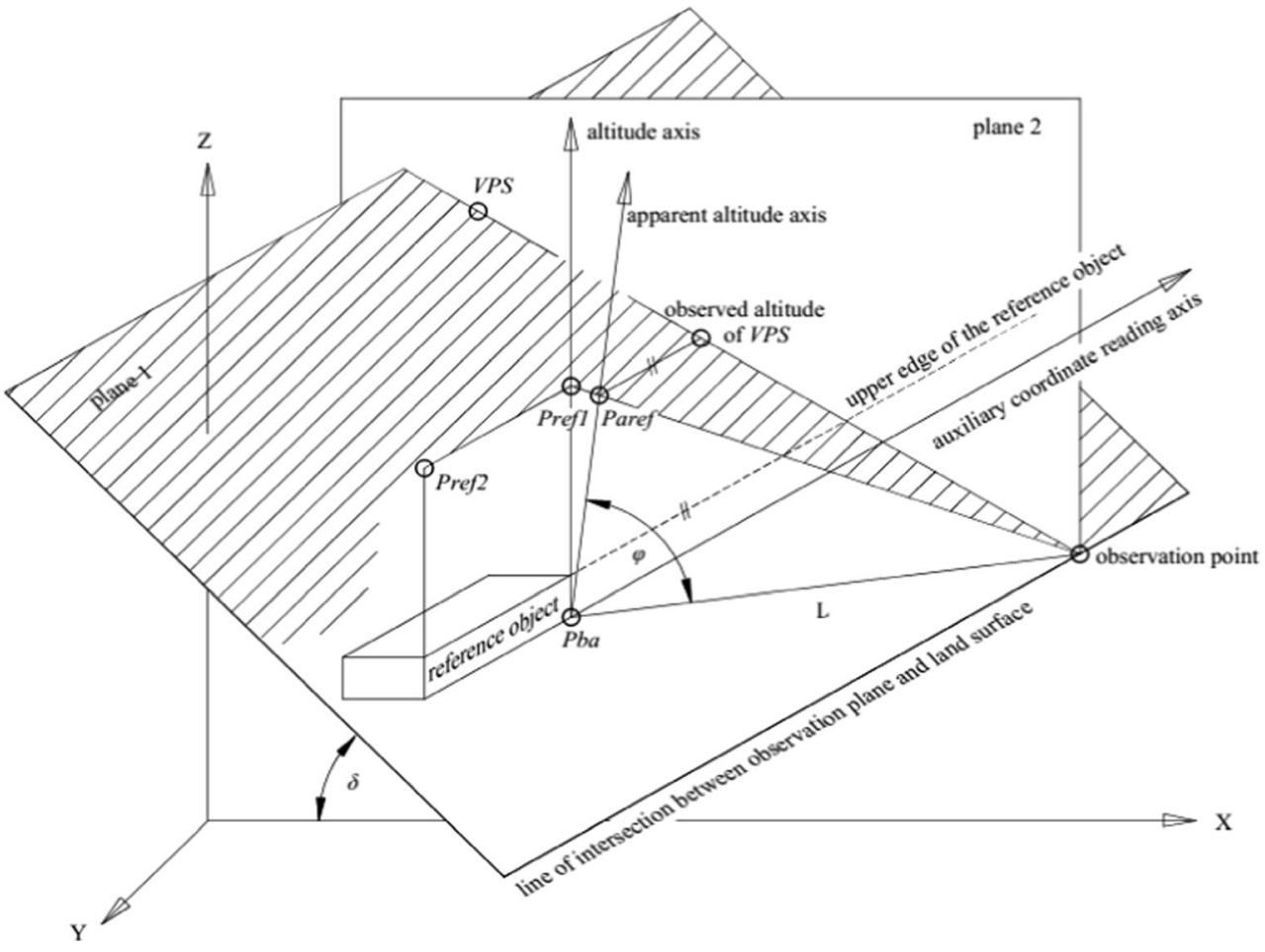

of this height visible on photograph. This axis is perpendicular to the direction of the observer's perception, i.e. diagonal towards the land surface. The angle $\varphi$ of its inclination towards the land surface changes depending on the VPS altitude and a known distance $L$ of the altitude axis from the observer. In the same way, a scale of length of the axis departs from the actual altitude scale depending on the VPS position over the horizon and distance $L$.

In order to read VPS altitude on the apparent altitude axis, projection of the VPS on this axis along the plane 1 is performed, what ends in finding point Paref. Next, the apparent altitude of the VPS must be converted into altitude of reference point Pref1 assigned to the altitude axis. It allows one to define plane 1 by establishing the position of the third point generating this plane (point Pref2 of altitude identical as point Pref1 and remaining coordinates being read from map for the second corner of the same object). Consequently, plane 1 crosses points Pref1, Pref2 and Pobs and intersects with the land surface along the straight line parallel to the edges of the reference object.

Ultimately, altitude Zref of the point at which the altitude axis intersects with plane 1 is obtained by:

- Projecting the VPS being observed on the apparent altitude axis in a direction parallel to the horizontal edge of the reference object based on the plume photograph (the reference object must be visible)point Paref

- Converting the Zaref apparent altitude read for point Paref into altitude Zref of point Prefl (length of the Pba_Prefl section) (Fig. 3).
Altitude Zref is calculated based on the following dependence:

Zref $=L \cdot \tan \left(2 \cdot \arcsin \frac{\text { Zaref }}{2 \cdot L}\right), \mathrm{m}$

where

$L \quad$ horizontal distance of the altitude axis from the observer, $\mathrm{m}$

Zaref apparent altitude, $\mathrm{m}$

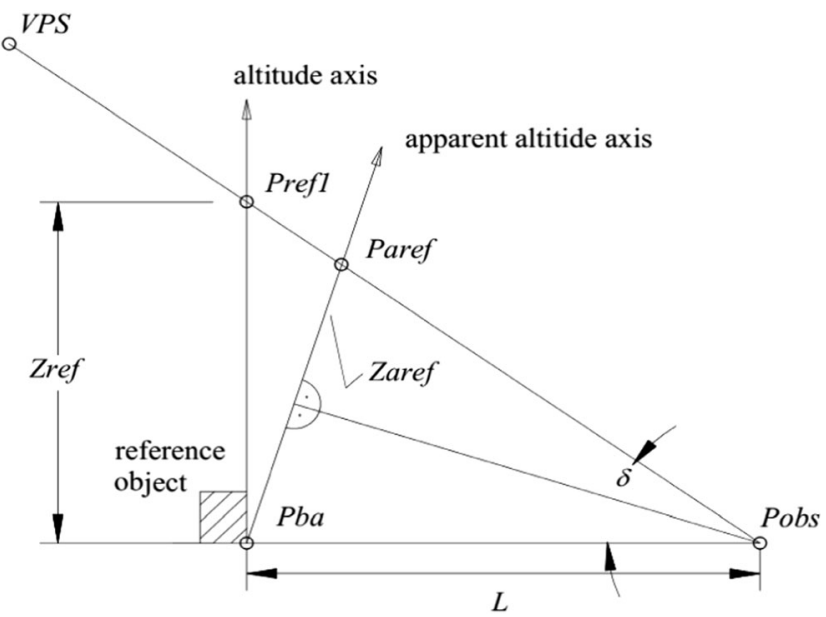

Fig. 3 Determining the altitude of intersection of plane 1 with the altitude axis 
The foregoing formula makes it possible to account for the sphericity of the observer's perception, by substituting a sector of a vertical circle having the centre at point Pobs, crossing points $P b a$ and Paref (as perceived by the observer), with section Pba_Paref, and then by converting its length into height Pba_Pref1.

Having known coordinates $x$ and $y$ of points Pref1, Pref2 and observation point Pobs (from map) and obtaining from Eq. (2) altitude Zref common to both points Pref1 and Pref2 allows one to find $D_{1}$ and establish the equation of plane 1 .

\subsection{Plane 2}

Plane 2 is a vertical plane of the observer's sight perpendicular to the land surface, crossing observation point Pobs (Fig. 4). In order to develop its mathematical description, one must find any easily recognisable object situated in this plane exactly under the VPS being observed. Next, on the map, it base is to be connected by means of a straight line with observation point Pobs (this line is formed by the points common for plane 2 and the surface of the earth). An intersection between this line and the auxiliary coordinates reading axis, running along the lower edge of the reference building, indicates coordinates $x$ and $y$ of points $P 2 b a$ and $P 2 e l$ lying on plane 2, one which is being sought. Their ordinates are 0 and Zref, respectively. Having learnt the full coordinates of three points generating plane 2 ( $P 2 b a, P 2 e l$ and Pobs), one can establish an equation of this plane.

\subsection{Plane 3}

Plane 3 is a vertical plane intersecting with the actual emission point $P e m$, perpendicular to the land surface, in which the plume moves in a direction corresponding to the wind vector

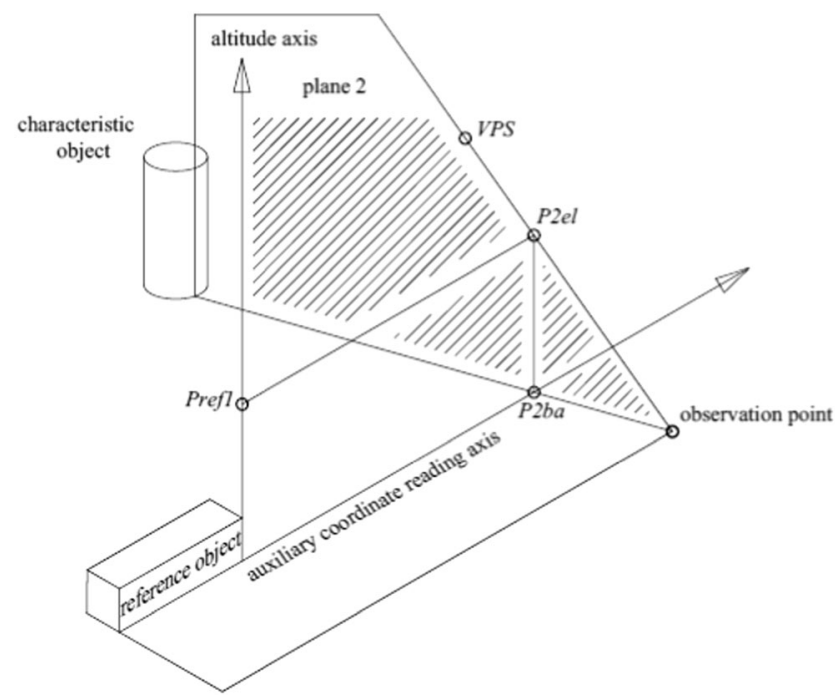

Fig. 4 Orientation of plane 2
(Fig. 1). The wind vector used for the purpose of plane 3 definition, is vectorially averaged in the period of time required by the plume to travel the path from the emission point to the VPS being observed. Then it is decomposed into components parallel to axes $X$ and $Y$ of the applied coordinate system $[8,9]$ :

$u_{x}=u_{o} \cdot \sin \alpha$

$u_{y}=u_{o} \cdot \cos \alpha$,

where

$u_{x} \quad u_{y}$, components of wind velocity vector $u_{o}$ in the direction of $X$ and $Y, \mathrm{~m} / \mathrm{s}$

$\alpha \quad$ wind azimuth - angle between the wind vector and the northern direction, deg.

After decomposition, the orientation of plane 3 does not depend on the wind velocity; hence, the constant wind velocity can be assumed to equal $1 \mathrm{~m} / \mathrm{s}$. The wind azimuth in Eq. (3) is obtained based on wind direction $\beta$ (in degrees), as follows:

$\alpha=\left\{\begin{array}{l}\beta-180^{\circ} \Leftrightarrow \beta>180^{\circ} \\ \beta+180^{\circ} \Leftrightarrow \beta \leq 180^{\circ}\end{array}\right.$

Therefore, one of the vectors lying in plane 3 is the horizontal vector of the wind direction: $e(\sin \alpha, \cos \alpha, 0)$. The second vector which may be always assigned to vertical plane 3 is a vertical unit vector: $k(0,0,1)$. Based on the vector product of the two vectors described above, one may find components of a vector normal towards plane 3 , and using the information on coordinates of the actual emission point, one may also establish the equation of this plane.

After determining equations describing planes $1,2,3$ on account of variables $x, y, z$, one can calculate coordinates of the VPS being sought by solving a system of three equations of the $A_{n} x+B_{n} y+C_{n} z=D_{n}$ type.

\subsection{Exemplary Calculations of the VPS Altitude}

The sample calculations of the VPS altitude have been conducted for the plume shown in the photograph (Fig. 5). In the first step, the VPS is projected on the apparent altitude axis in parallel to the upper edge or the characteristic object, thus obtaining point Paref. Next, using the map, on the base of references to the objects visible on the photograph, one determines the position of the point of intersection between plane 2 and the auxiliary axis, which reveals coordinates $x$ and $y$ of points $P 2 b a$ and $P 2 e l$. The map depicting the area studied is properly scaled and oriented towards the north (Fig. 6). This map is also used to find coordinates of points described in the equations of planes referred to. As an example, the values of subsequent variables, obtained during VPS height calculations, are shown in the Tables 1,2 and 3, with corresponding uncertainties marked with $\mathrm{u}()$. 
Fig. 5 Determination of altitude Zaref being observed and the position of point $P 2 b a$

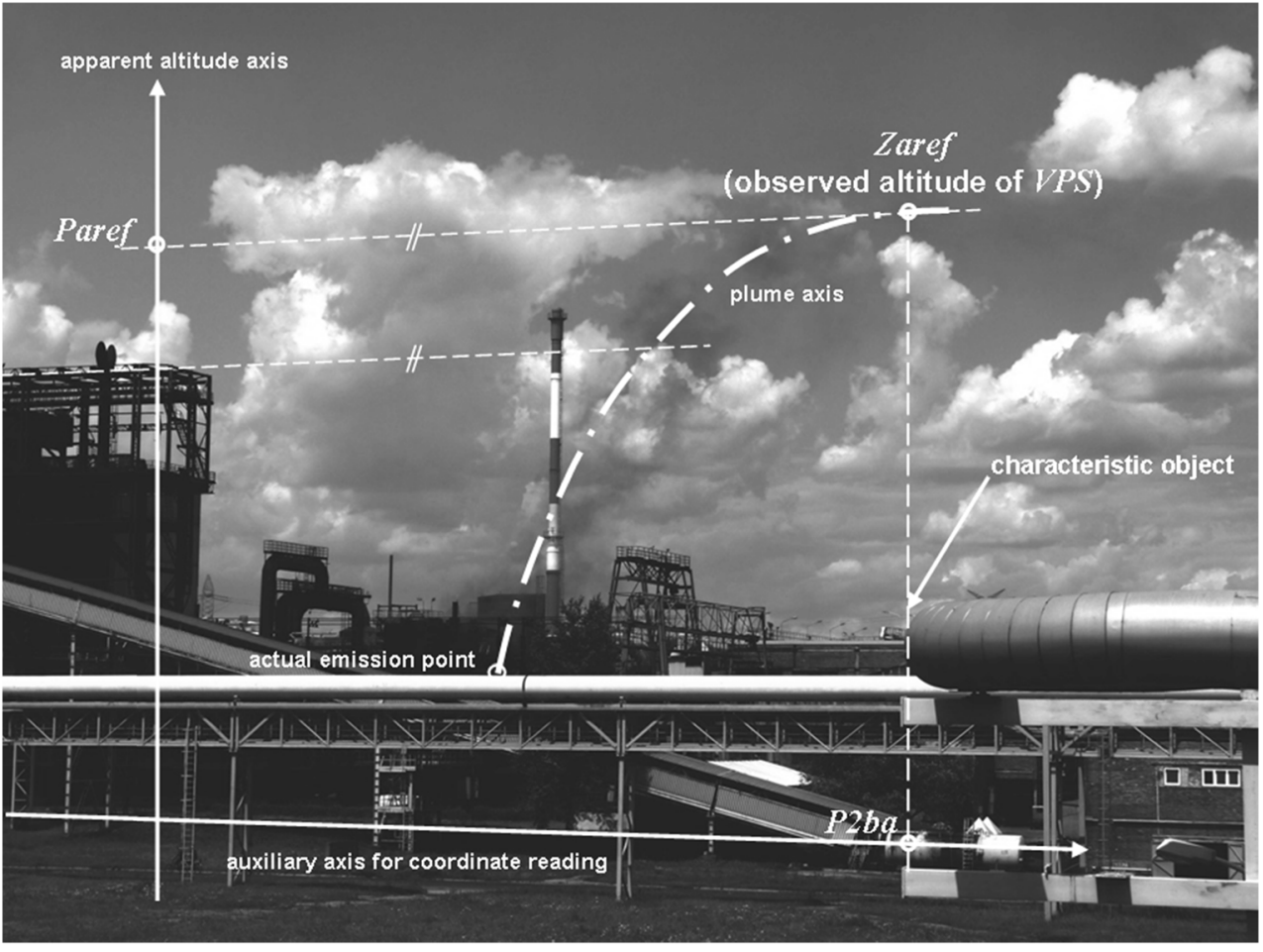

The coordinates being sought for the VPS positioned at the intersection of the aforementioned three planes are 2142, 8064 and $56 \mathrm{~m}$. Consequently, the virtual point emission source altitude calculated is $56 \mathrm{~m}$. The estimated uncertainty of VPS height assessment is $\pm 8.5 \mathrm{~m}$ (about $15 \%$ of the VPS height).

\section{Results and Discussion}

\subsection{Studies of Plume Rise Above a Bank of Coke Ovens}

By application of the methodology discussed, studies were conducted on the premises of one of Polish coking plants in order to determine the VPS altitude for different wind directions and velocities as well as atmosphere stabilities. They consisted in taking a series of more than 500 photographs of a trajectory of a waste gas plume having its emission point at the roof of a bank of coke ovens. The photographs were framed in a manner ensuring that they also included characteristic objects enabling the calculations discussed to be done. At the same time, measurements of the wind direction and velocity were conducted at the altitude of $10 \mathrm{~m}$, on a 10 -min time of vectorial averaging. In all cases analysed, this time was longer than the time between the emission and the moment when the plume reaches the VPS. The atmosphere stability was assessed based on the value of solar radiation by application of the solar radiation delta-T (SRDT) method [10,
11]. Having selected and contrasted the photographs using image processing software, and having added the apparent altitude axis and the auxiliary coordinate reading axis, one could ultimately use 84 of them to read the Zaref altitude being observed. The VPS altitudes (observed) obtained by the method discussed were collated with the altitudes calculated by application of formulas used when dealing with considerable heat emission, namely the Briggs formula $[1,12]$ and formulas envisaged for point and line sources applied under the BLP model [13].

The VPS thus obtained for the selected cases has been provided in Table 4. In the table, the uncertainties of VPS height calculations have been also presented. The accuracy of wind direction measurement used in uncertainty assessment, is assumed to be $2^{\circ}$ independent from wind direction.

Figure 7 provides a graph comparing VPS altitudes observed and calculated by application of the methods discussed.

The differences in evaluated VPS altitudes, incorporated into calculations, might entail significant variances in estimated concentration values and also in places where their maxima occur. In Table 5, maximum concentrations calculated for subsequent VPS altitudes presented in Table 4, along with distances of their occurrences from the emission source, are shown. Calculations were executed with use of simple point Gaussian dispersion model, with the assumed emission $1 \mathrm{~g} \cdot \mathrm{s}^{-1}$ of gaseous substance and ground roughness $z_{0}=1 \mathrm{~m}$. 
Fig. 6 Map used to establish coordinates for the purposes of calculations

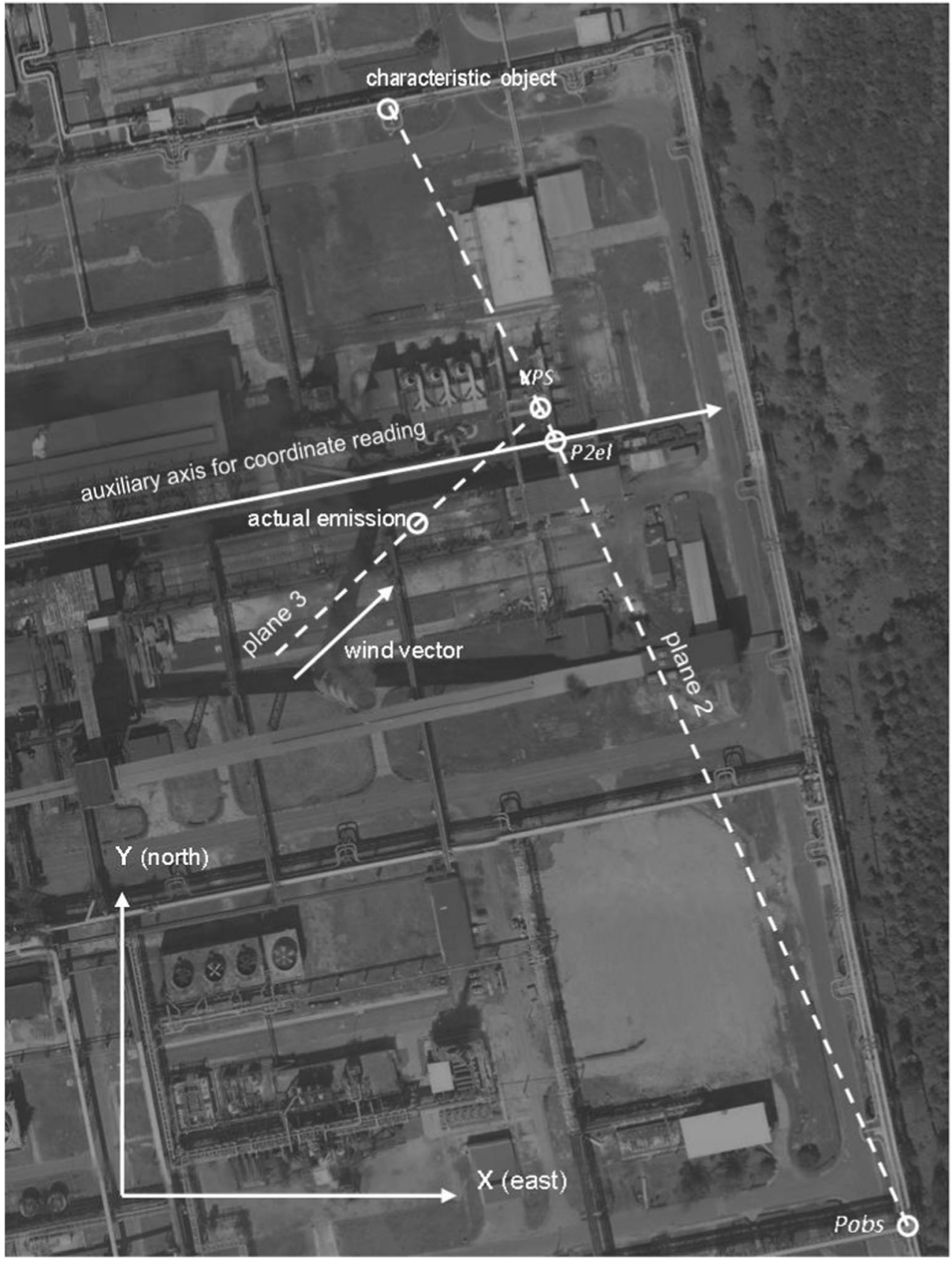

For the purpose of statistical assessment of conformity between the VPS altitudes observed and those calculated, specific statistical indicators were applied, namely $R M S$, being generally used in statistical error assessment [14, 15] and NMSE, commonly used for assessment of modelling quality [3].

The $R M S$ (root mean square) is calculated according to the following dependence:

$R M S=\sqrt{\frac{1}{n} \cdot \sum_{i=1}^{n}\left(H_{p i}-H_{o i}\right)^{2}}$, where

$H_{p i} \quad V P S$ altitude observed for the $i$ th case

$H_{o i} \quad V P S$ altitude calculated for the $i$ th case

Normalised mean square error NMSE is calculated in the following manner:

$$
N M S E=\frac{\frac{1}{n} \cdot \sum_{i=1}^{n}\left(H_{p i}-H_{o i}\right)^{2}}{\frac{1}{n^{2}} \cdot \sum_{i=1}^{n}\left(H_{p i} \cdot H_{o i}\right)} .
$$


Table 1 VPS altitude calculations - point coordinates and wind angle

\begin{tabular}{|c|c|c|c|c|c|c|c|c|}
\hline & \multicolumn{3}{|c|}{ Coordinates } & \multirow[b]{2}{*}{ Angle, deg } & \multicolumn{4}{|c|}{ Uncertainty } \\
\hline & $x, \mathrm{~m}$ & $y, \mathrm{~m}$ & $z, \mathrm{~m}$ & & $\mathrm{u}(x), \mathrm{m}$ & $\mathrm{u}(y), \mathrm{m}$ & $\mathrm{u}(z), \mathrm{m}$ & $\mathrm{u}(\alpha), \mathrm{rad}$ \\
\hline \multicolumn{9}{|c|}{ Points characterising the geometry of the reference system, read from the map (scale 1:500) } \\
\hline Pobs & 2255 & 7811 & 17 & - & 0.29 & 0.29 & 0.012 & \\
\hline$P b a$ & 2064 & 8038 & 0 & - & 0.29 & 0.29 & 0.012 & \\
\hline Pref1 & 2064 & 8038 & Zref & - & 0.29 & 0.29 & 0.70 & \\
\hline Pref2 & 1948 & 8019 & Zref & - & 0.29 & 0.29 & 0.70 & \\
\hline Pem & 2107 & 8031 & 11 & - & 0.29 & 0.29 & 0.012 & \\
\hline $\begin{array}{l}\text { height of reference object } \\
\text { (from project) }\end{array}$ & - & - & 43 & - & - & - & 0.012 & \\
\hline \multicolumn{9}{|c|}{ Observation data extracted from the photograph and map } \\
\hline Zaref & - & - & 53 & - & - & - & 0.65 & \\
\hline$P 2 b a$ & 2146 & 8056 & 0 & - & 0.29 & 0.29 & 0.012 & \\
\hline$P 2 e l$ & 2146 & 8056 & Zref & - & 0.29 & 0.29 & 0.70 & \\
\hline \multicolumn{9}{|l|}{ Measurement data } \\
\hline$\alpha$ & - & - & - & 47 & - & - & - & 0.010 \\
\hline \multicolumn{9}{|l|}{ Computational values } \\
\hline Zref & - & - & 54 & - & - & - & 0.70 & \\
\hline
\end{tabular}

Table 6 contains results of the assessment of statistical conformity between the calculated VPS altitude and the observed one.

\section{Discussion of the Results Obtained}

Having analysed the relevant graphs as well as the $R M S$ and NMSE values, one may find the altitudes obtained by application of the Briggs and the point source BLP methods highly conforming with the altitude observed. The conformity in question is slightly higher when the point source BLP method is applied, as confirmed by

Table 2 VPS altitude calculations - components $\Delta x, \Delta y$ and $\Delta z$ of particular plane's vectors

\begin{tabular}{llll}
\hline & Component & & \\
\cline { 2 - 4 } & $\Delta x \pm \mathrm{u}(\Delta x), \mathrm{m}$ & $\Delta y \pm \mathrm{u}(\Delta y), \mathrm{m}$ & $\Delta z \pm \mathrm{u}(\Delta z), \mathrm{m}$ \\
\hline Plane 1 & & & \\
Vector 1 & $-191.00 \pm 0.41$ & $227.00 \pm 0.41$ & $37.00 \pm 0.70$ \\
Vector 2 & $-307.00 \pm 0.41$ & $208.00 \pm 0.41$ & $37.00 \pm 0.70$ \\
Plane 2 & & & \\
Vector 1 & $-109.00 \pm 0.41$ & $245.00 \pm 0.41$ & $-17.00 \pm 0.012$ \\
Vector 2 & $-109.00 \pm 0.41$ & $245.00 \pm 0.41$ & $37.00 \pm 0.70$ \\
Plane 3 & & & \\
Vector 1 & $0.00 \pm 0.00$ & $0.00 \pm 0.00$ & $1.00 \pm 0.00$ \\
Vector 2 & $0.7310 \pm 0.0070$ & $0.6820 \pm 0.0070$ & $0.00 \pm 0.00$ \\
\hline
\end{tabular}

the NMSE coefficient; however, its value obtained in both cases analysed is lower than one considered to be acceptable, i.e. $1.5[16,17]$, with the $R M S$ values being very close to one another. The proximity of results obtained by application of the said two methods stems from the fact that an extended and modified Briggs algorithm was used under the point and also line source BLP methods. For this reason, as the source length approaches zero, the line source plume rise equations reduce to the BLP point source equations. In turn, these equations reduce to Briggs point source plume rise equations when vertical wind shear and downwash are neglected [13]. The further analysis shows that the line source BLP method implies too small VPS altitudes for a bank of coke ovens, which is reflected by the NMSE value exceeding 1.5. The most likely reason, why BLP line source method results in calculations deviating from results obtained from point model, is that BLP line model has been developed for aluminium reduction plants with particular emphasis on aluminium reduction facility potrooms, which create line sources with a very high unit emission of heat. Pootroms are usually configured as multiply rows of closely spaced line sources, which is a typical configuration for aluminium reduction plants. In this case neighbouring line sources generate adjacent plumes tending to block entrainment of the ambient air, which maintains the energy of convection. In this way every single source influences the buoyancy effect generated by the whole group of sources. This results in enhancement of plume rise over that revealed by isolated line source plume-under the condition that 
Table 3 VPS altitude calculations - coefficients $A_{n}, B_{n}$, $C_{n}$ and $D_{n}$ of subsequent planes

\begin{tabular}{lllll}
\hline & Coefficient & & & \\
\cline { 2 - 5 } & $A_{n} \pm \mathrm{u}\left(A_{n}\right), \mathrm{m}$ & $B_{n} \pm \mathrm{u}\left(B_{n}\right), \mathrm{m}$ & $C_{n} \pm \mathrm{u}\left(C_{n}\right), \mathrm{m}$ & $D_{n} \pm \mathrm{u}\left(D_{n}\right), \mathrm{m}$ \\
\hline Plane 1 & $696.00 \pm 0.78$ & $-4251.00 \pm 0.78$ & $29,961.00 \pm 0.58$ & $-31,120,000 \pm 1300$ \\
Plane 2 & $13,143.00 \pm 0.78$ & $5847.00 \pm 0.78$ & $0.00 \pm 0.58$ & $75,310,000 \pm 4100$ \\
Plane 3 & $0.6820 \pm 0.0070$ & $-0.7310 \pm 0.0070$ & $0.0000 \pm 0.0070$ & $-4437.00 \pm 0.29$ \\
\hline
\end{tabular}

other neighbouring sources are present. If not, the formula covering this phenomenon, applied for one only sources row with a far lower temperature (as the banks of coke oven in compare to the target objects-rows of pootroms), tends to create lower values of rise. The next reason might be the fact that the whole energy of buoyancy, instead of being concentrated at one point as in a case of point source, is spread along the centerline of the sources row. It increases the surface of heat loss from convective stream, resulting in abatement of the plume rise. Another factor is that the effective length of a particular line source depends on the relations between wind direction and direction of the sources row centerline. This creates relationship in which the plume rises lower when the vector of wind mean velocity is perpendicular to the long axis of the source and higher when the wind direction is parallel to this line. The BLP calculation formula forces a rapid drop of rise as the result of relatively slight wind vector deviation from the direction of sources row axis, ending in minimal value at the angle $90^{\circ}$. This was observed during calculations, when the angle between wind vector and the banks of coke oven long axis varied in vast range. Location point of observed plume on the source surface is also important - the closer to the windward end of the source it is located, the greater part of the heat released from the source has an impact on the plume elevation and thus greater VPS height is noticed.

Other phenomena characteristic for low sources, like transitional plume rise and building downwash are not important for taller stacks and are not treated by standard Briggs equations. In turn, they are usually taken into account in calculations for line sources, where mentioned phenomena are vital. They were also included in presented here plume rise calculations for point sources as well as line ones.

Taking into account the methodology of measurement, presented method can be used in all weather conditions, except these characterised by a very weak overall visibility (darkness, rain, snow). In reality, some conditions make it extremely difficult or even impossible for use. It includes strong wind and weak stability of the atmosphere. Especially the first case, reducing the concentration of contaminants in the plume and thereby limiting its visibility creates difficult conditions for measurement. In a case of the instability of the atmosphere, irregularly shaped plume of looping character appears. If it is good visibly, one can specify its axis, making an assumption that it lies midway between the upper and lower plume border,

Table 4 Collation of sample VPS altitudes

\begin{tabular}{|c|c|c|c|c|c|c|c|c|}
\hline \multirow[t]{2}{*}{ Observation date } & \multicolumn{4}{|c|}{ Meteorological parameters } & \multicolumn{4}{|c|}{$V P S$ altitude determined according to the following methods, $\mathrm{m}$} \\
\hline & $\begin{array}{l}\text { Wind velocity, } \\
\mathrm{m} \mathrm{s}^{-1}\end{array}$ & $\begin{array}{l}\text { Wind direction, } \\
\text { deg }\end{array}$ & $\begin{array}{l}\text { Radiation, } \\
\mathrm{W} \mathrm{m}^{-2}\end{array}$ & $\begin{array}{l}\text { Atmosphere } \\
\text { stability }\end{array}$ & $\begin{array}{l}\text { Presented } \\
\text { method, m }\end{array}$ & $\begin{array}{l}\text { BLP line } \\
\text { source, } m\end{array}$ & Briggs, $\mathrm{m}$ & $\begin{array}{l}\text { BLP point } \\
\text { source, } m\end{array}$ \\
\hline 9 May 2012 & 1.27 & 235 & 720 & A & $68 \pm 10$ & 33.0 & 43.1 & 42.9 \\
\hline 10 May 2012 & 0.64 & 220 & 170 & $\mathrm{D}$ & $63.0 \pm 9.5$ & 32.0 & 69.8 & 60.5 \\
\hline 10 May 2012 & 1.56 & 210 & 517 & $\mathrm{~B}$ & $44.0 \pm 6.6$ & 32.9 & 46.9 & 44.0 \\
\hline 11 May 2012 & 0.82 & 239 & 162 & $\mathrm{D}$ & $43.0 \pm 6.5$ & 25.9 & 50.8 & 46.5 \\
\hline 11 May 2012 & 2.36 & 222 & 530 & $\mathrm{C}$ & $37.0 \pm 5.6$ & 15.4 & 26.1 & 26.5 \\
\hline 15 May 2012 & 1.02 & 88 & 102 & $\mathrm{D}$ & $36.0 \pm 5.4$ & 47.5 & 74.8 & 64.1 \\
\hline 23 May 2012 & 1.06 & 73 & 191 & $\mathrm{~B}$ & $19.0 \pm 2.9$ & 33.5 & 49.2 & 45.9 \\
\hline 23 May 2012 & 1.76 & 82 & 718 & A & $30.0 \pm 4.5$ & 11.0 & 23.3 & 23.6 \\
\hline 25 May 2012 & 1.19 & 30 & 213 & $\mathrm{~B}$ & $37.0 \pm 5.6$ & 11.0 & 33.7 & 33.1 \\
\hline 25 May 2012 & 1.38 & 95 & 487 & $\mathrm{~B}$ & $37.0 \pm 5.6$ & 12.2 & 30.4 & 30.2 \\
\hline
\end{tabular}


Fig. 7 Comparison of VPS altitudes observed and calculated by the methods discussed

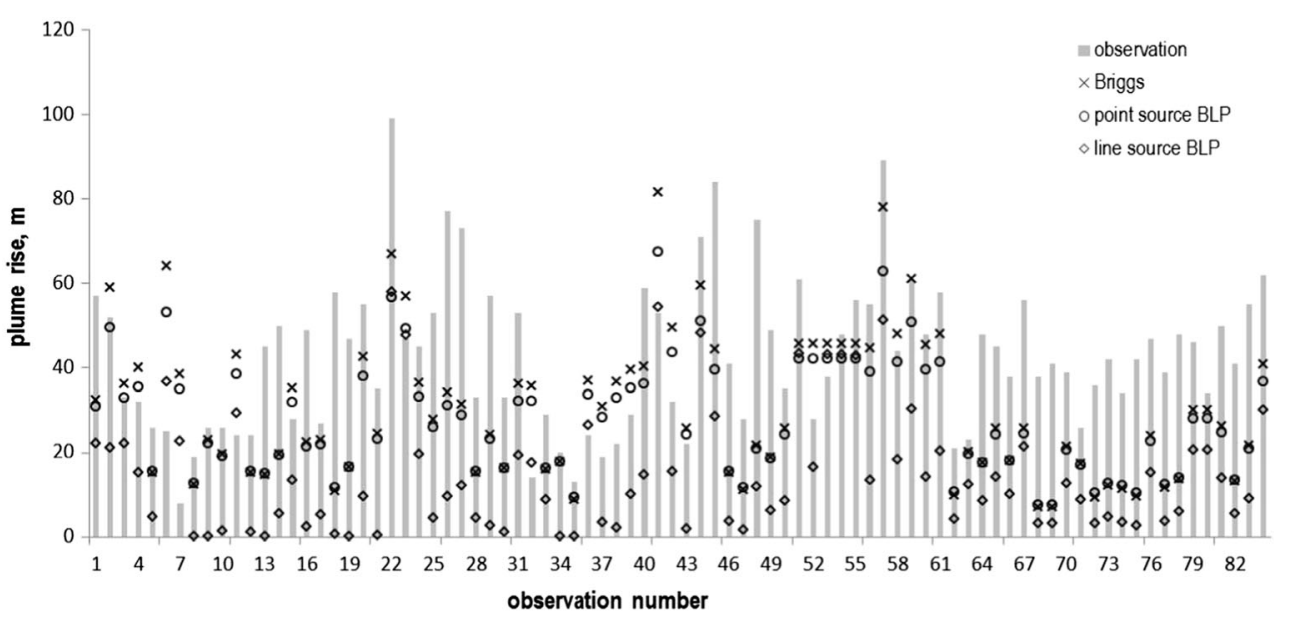

which is usually relatively well contrasted with the surroundings.

In most cases, concentrations achieved with the use of the presented observational method for calculating the VPS height are lower than the levels obtained using popular calculation algorithms. However, there are a number of cases where the opposite is true - the levels achieved with the observed VPS heights surpass those obtained with VPS calculated. These cases are particularly important - they mean an increased risk for the environment due to the lack of suitable control over air purity while standard calculations are employed. The method described here can be used as a simple tool for a partial, quick correctness control, carried out in order to detect and eliminate underestimations in the standard calculations of concentrations.

\section{Conclusion}

Using commonly available and cheap technical means, one is able to assess the value of plume rise from different emitters in a more accurate manner compared to computational methods. The increase of VPS height valuation reliability is vital due to its high influence on concentration

In order to increase the reliability of assessment of plume rise values, it is a prerequisite that the method proposed should be applied multiple times under comparable meteorological conditions, which makes it possible to minimise errors in statistical terms. The method discussed may also be used to test computational models of plume rise, particularly in cases of nontypical emission sources. This method requires that the plume should be clearly contrasted against the background (i.e. the sky) which, in many situations, necessitates application of highly efficient smoke generators, fluorescent substances or smoke candles. One may also use thermal imaging cameras at night, assuming that both the plume and the reference objects are clearly visible, which would require a sufficient difference between their temperatures and that of the surrounding air.

Table 5 Maximum concentrations $C_{m}$ and distances of their occurrence $x_{m}$, calculated for obtained altitudes $V P S$

\begin{tabular}{|c|c|c|c|c|c|c|c|c|c|c|}
\hline \multirow[t]{2}{*}{ Observation date } & \multicolumn{2}{|c|}{ Meteorological parameters } & \multicolumn{2}{|c|}{ Presented method } & \multicolumn{2}{|c|}{ BLP line source } & \multicolumn{2}{|l|}{ Briggs } & \multicolumn{2}{|c|}{ BLP point source } \\
\hline & $\begin{array}{l}\text { Wind velocity, } \\
\mathrm{m} \mathrm{s}^{-1}\end{array}$ & $\begin{array}{l}\text { Atmos. } \\
\text { stability }\end{array}$ & $C_{m}, \mu \mathrm{g} \mathrm{m}^{-3}$ & $x_{m}, \mathrm{~m}$ & $C_{m}, \mu \mathrm{g} \mathrm{m}^{-3}$ & $x_{m}, \mathrm{~m}$ & $C_{m}, \mu \mathrm{g} \mathrm{m}^{-3}$ & $x_{m}, \mathrm{~m}$ & $C_{m}, \mu \mathrm{g} \mathrm{m}^{-3}$ & $x_{m}, \mathrm{~m}$ \\
\hline 9 May 2012 & 1.27 & A & 20.71 & 186 & 76.93 & 94 & 47.44 & 121 & 47.84 & 120 \\
\hline 10 May 2012 & 0.64 & $\mathrm{D}$ & 39.36 & 413 & 187.44 & 153 & 31.06 & 481 & 43.22 & 389 \\
\hline 10 May 2012 & 1.56 & B & 45.26 & 131 & 79.90 & 95 & 39.94 & 140 & 45.26 & 131 \\
\hline 11 May 2012 & 0.82 & $\mathrm{D}$ & 74.12 & 235 & 237.80 & 113 & 50.48 & 300 & 61.89 & 163 \\
\hline 11 May 2012 & 2.36 & $\mathrm{C}$ & 42.06 & 130 & 261.60 & 45 & 87.16 & 85 & 84.46 & 86 \\
\hline 15 May 2012 & 1.02 & $\mathrm{D}$ & 89.70 & 182 & 47.37 & 272 & 16.61 & 533 & 23.73 & 423 \\
\hline 23 May 2012 & 1.06 & $\mathrm{~B}$ & 342.90 & 53 & 113.5 & 97 & 53.52 & 148 & 61.31 & 137 \\
\hline 23 May 2012 & 1.76 & A & 65.94 & 86 & 401.23 & 34 & 104.05 & 68 & 101.68 & 69 \\
\hline 25 May 2012 & 1.19 & B & 83.26 & 108 & 883.55 & 30 & 99.94 & 98 & 103.50 & 96 \\
\hline 25 May 2012 & 1.38 & $\mathrm{~B}$ & 71.80 & 108 & 623.16 & 33 & 105.38 & 88 & 106.75 & 87 \\
\hline
\end{tabular}


Table $6 \quad R M S$ and NMSE values corresponding to the calculation methods acpplied to determine the VPS altitude

Method applied to calculate the apparent emission point altitude

\begin{tabular}{llll}
\cline { 2 - 4 } & Line source BLP & Briggs method & Point source BLP \\
\hline RMS & 32.68 & 22.38 & 22.68 \\
NMSE & 1.74 & 0.44 & 0.39 \\
\hline
\end{tabular}

Acknowledgments The paper has been made within the framework of project No. POIG.01.01.02-24-017/08 "Smart Coke Plant Meeting the Requirements of Best Available Techniques", which was co-financed by the European Regional Development Fund (ERDF).

Open Access This article is distributed under the terms of the Creative Commons Attribution 4.0 International License (http:// creativecommons.org/licenses/by/4.0/), which permits unrestricted use, distribution, and reproduction in any medium, provided you give appropriate credit to the original author(s) and the source, provide a link to the Creative Commons license, and indicate if changes were made.

\section{References}

1. Briggs, G. A. (1984). Plume rise and buoyancy effects. In Atmospheric Science and Power (pp. 327-366). D. Randerson ed., US Department of Energy.

2. Turner, B. D. (1994). Workbook of Atmospheric Dispersion Estimates: an Introduction to Dispersion Modelling (2nd ed.). Boca Raton: Lewis Publishers.

3. Markiewicz, M. T. (2004). Podstawy modelowania rozprzestrzeniania sie zanieczyszczeń w powietrzu atmosferycznym. Warszawa: Oficyna Wydawnicza PW.
4. Zanetti, P. (1990). Air Pollution Modelling: Theories, Computational Methods and Available Software. New York: Van Nostrand-Reinhold.

5. Flir, G. F. (2009). 320 User Manual. North Billerica: FLIR Systems USA Thermography Center.

6. Kajetanowicz, P., \& Wierzejewski, J. (2008). Algebra z geometria analityczna. Warszawa: PWN.

7. Radziszewski, Z. (2010). Geometria analityczna. Lublin: UMCS.

8. EPA. (2000). Meteorological Monitoring Guidance for Regulatory Modeling Applications EPA-454/R-99-005. Research Triangle Park: U.S. EPA.

9. Seinfeld, J. H., \& Pandis, S. N. (2006). Atmospheric Chemistry and Physics: from Air Pollution to Climate Change (2nd ed.). Hoboken: John Wiley and Sons Inc.

10. Bowen, B. M., Dewart, J. M., \& Chen, A. I. (1983). Stability class determination: a comparison for one site. In Proceedings of the Sixth Symposium on Turbulence and Diffusion (pp. 211-214). Boston: American Meteorological Society.

11. EPA. (1994). An Evaluation of a Solar Radiation Delta-T Method for Estimating Pasquill-Gifford (P-G) Stability Categories, EPA-454/R-93-055. Research Triangle Park: U.S. EPA.

12. Briggs, G. A. (1975). Plume rise predictions. In Lectures on Air Pollution and Environmental Impact Analyses. Worksop Proceedings (pp. 59-111). Boston.

13. Schulman, L., \& Scire, J. S. (1980). Buoyant line and point source $(B L P)$ dispersion model user's guide. Concord: Environmental Research and Technology.

14. Hill, T., \& Lewicki, P. (2007). Statistics: methods and applications. Tulsa: StatSoft.

15. Bobrowski, D. (1986). Probabilistyka w zastosowaniach technicznych. Warszawa: WNT.

16. Chang, J. C., \& Hanna, S. R. (2004). Air quality model performance evaluation. Meteorology and Atmospheric Physics, 87, 167-196.

17. Chang, J. C., \& Hanna, S. R. (2005). Technical Descriptions and User's Guide for the BOOT Statistical Model Evaluation Software Package. 